\title{
Fragment-Based Covalent Ligand Screening Enables Rapid Discovery of Inhibitors for the RBR E3 Ubiquitin Ligase HOIP
}

\author{
Henrik Johansson, ${ }^{\dagger, \ddagger}$ Yi-Chun Isabella Tsai, ${ }^{\ddagger}$ Ken Fantom, ${ }^{\S}$ Chun-Wa Chung, ${ }^{\dagger, \S}$ Sandra Kümper, ${ }^{\dagger, \ddagger}$ \\ Luigi Martino, ${ }^{*}$ Daniel A. Thomas, ${ }^{\S}$ H. Christian Eberl, ${ }^{\|}$Marcel Muelbaier, ${ }^{\| \odot}$ David House, ${ }^{*}{ }^{\dagger}(0)$ \\ and Katrin Rittinger*, ${ }^{*}+$ (D) \\ ${ }^{\dagger}$ Crick-GSK Biomedical LinkLabs, GlaxoSmithKline, Gunnels Wood Road, Stevenage SG1 2NY, United Kingdom \\ ${ }^{\ddagger}$ Molecular Structure of Cell Signalling Laboratory, The Francis Crick Institute, 1 Midland Road, London NW1 1AT, United \\ Kingdom \\ ${ }^{\S}$ R\&D Platform Technology \& Science, GlaxoSmithKline, Gunnels Wood Road, Stevenage SG1 2NY, United Kingdom \\ "Cellzome GmbH, a GlaxoSmithKline Company, Meyerhofstraße 1, Heidelberg 69117, Germany
}

Supporting Information

\begin{abstract}
Modification of proteins with polyubiquitin chains is a key regulatory mechanism to control cellular behavior and alterations in the ubiquitin system are linked to many diseases. Linear (M1-linked) polyubiquitin chains play pivotal roles in several cellular signaling pathways mediating immune and inflammatory responses and apoptotic cell death. These chains are formed by the linear ubiquitin chain assembly complex (LUBAC), a multiprotein E3 ligase that consists of 3 subunits, HOIP, HOIL-1L, and SHARPIN.

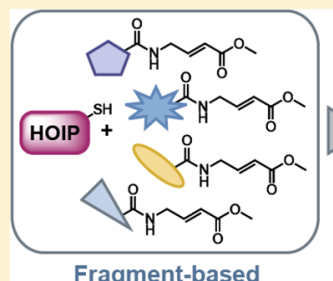

Fragment-based covalent ligand screening

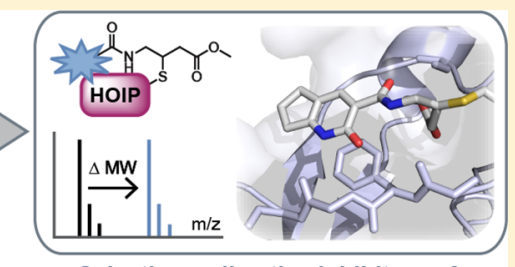

Selective, cell-active inhibitors of HOIP RBR E3 ligase

Herein, we describe the discovery of inhibitors targeting the active site cysteine of the catalytic subunit HOIP using fragmentbased covalent ligand screening. We report the synthesis of a diverse library of electrophilic fragments and demonstrate an integrated use of protein LC-MS, biochemical ubiquitination assays, chemical synthesis, and protein crystallography to enable the first structure-based development of covalent inhibitors for an RBR E3 ligase. Furthermore, using cell-based assays and chemoproteomics, we demonstrate that these compounds effectively penetrate mammalian cells to label and inhibit HOIP and $\mathrm{NF}-\kappa \mathrm{B}$ activation, making them suitable hits for the development of selective probes to study LUBAC biology. Our results illustrate the power of fragment-based covalent ligand screening to discover lead compounds for challenging targets, which holds promise to be a general approach for the development of cell-permeable inhibitors of thioester-forming E3 ubiquitin ligases.
\end{abstract}

\section{INTRODUCTION}

Ubiquitination represents one of the most diverse posttranslational modifications of proteins and constitutes an essential route for the regulation of protein signaling and degradation. The process is carried out through a complex interplay of enzymes (E1 ubiquitin-activating enzymes, E2 ubiquitin-conjugating enzymes, E3 ubiquitin ligases, and deubiquitinating (DUB) enzymes), which provides an intricate network of regulation and substrate specificity (Figure 1A). ${ }^{1,2}$ E3 ubiquitin ligases are the key determinants for substrate specificity in this cascade and as such represent attractive yet notoriously challenging pharmacological targets. There are over 600 known human E3 ligases that fall into three main classes (RING, HECT, and RBR) based on their structure and mechanism of ubiquitin transfer, which in the case of HECT and RBR E3 ligases involves the formation of a covalent thioester intermediate with ubiquitin. ${ }^{3,4}$ However, a deeper understanding of their underlying biology has long been hampered by the lack of selective pharmacological tools. The linear ubiquitin chain assembly complex (LUBAC) is a multiprotein E3 ubiquitin ligase of the RBR family that catalyzes the formation of polyubiquitin chains linked between the C-terminal carboxylate of ubiquitin and the N-terminal $\alpha$ amino group of methionine 1, called linear or M1-linked chains. ${ }^{5,6}$ Linear polyubiquitin chains play crucial roles in the regulation of multiple cellular functions including immune and inflammatory signaling via the NF- $\kappa \mathrm{B}$ pathway, cell death, and cancer. $^{7-10}$ LUBAC consists of three core components, the RBR-domain containing subunits HOIP and HOIL-1L plus SHARPIN, ${ }^{11-13}$ with HOIP constituting the catalytic machinery required for linear chain formation (Figure 1B). ${ }^{14-16}$ Recent crystal structures of truncated HOIP have provided valuable insight into the mechanism of linear polyubiquitin chain formation. ${ }^{15,17}$ However, in order to interrogate the physiological function of LUBAC in a cellular context, chemical tools that selectively target the E3 ligase activity of HOIP would be of great value. To this end, a handful of HOIP

Received: December 10, 2018

Published: January 18, 2019 
A

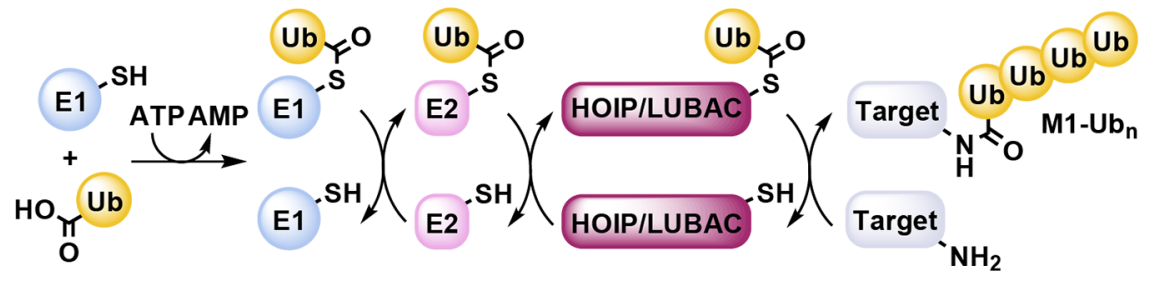

B HOIP

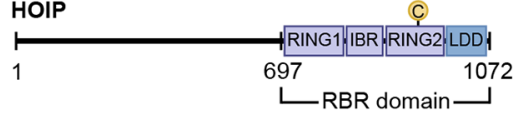

C

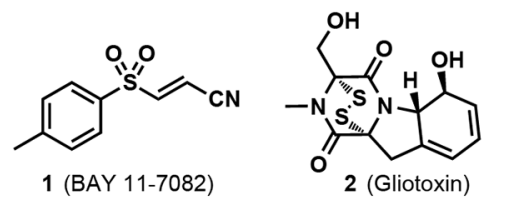

D

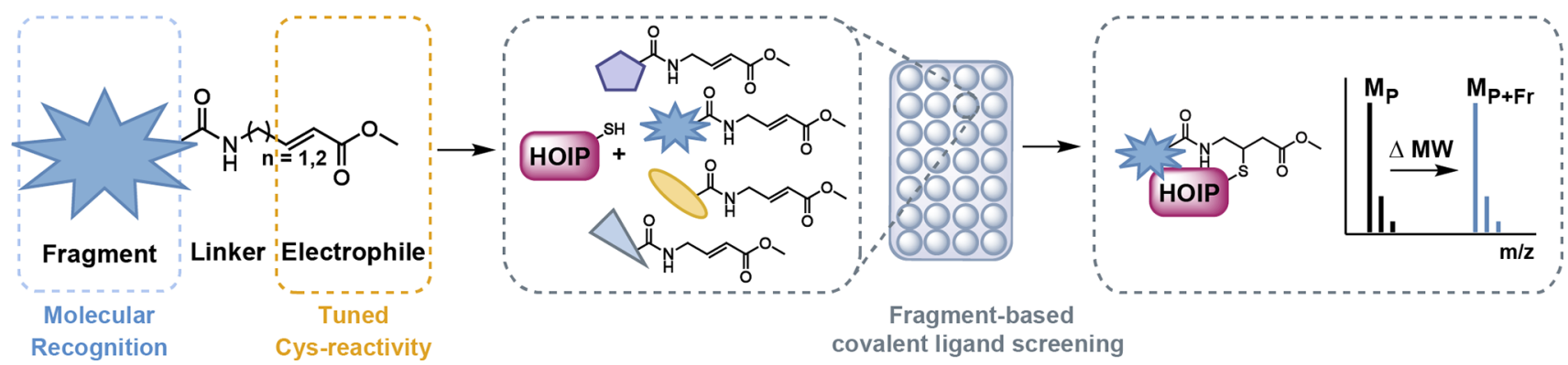

Figure 1. Targeting HOIP using fragment-based covalent ligand screening. (A) Schematic of the ubiquitination cascade highlighting the formation of linear (M1) polyubiquitin chains $\left(\mathrm{Ub}_{\mathrm{n}}\right)$ by LUBAC, a reaction that proceeds via a covalent thioester intermediate between the HOIP subunit and ubiquitin. (B) Schematic representation of the HOIP E3 ligase, highlighting the RBR which contains the active-site cysteine residue C885 (yellow circle) and the linear chain-determining domain, LDD (amino acids 697-1072). (C) Small molecule LUBAC inhibitors (1) BAY 11-7082 and (2) gliotoxin. (D) Overview of our approach to develop covalent probes targeting the active site cysteine residue of HOIP using fragmentbased covalent ligand screening by protein LC-MS.

A

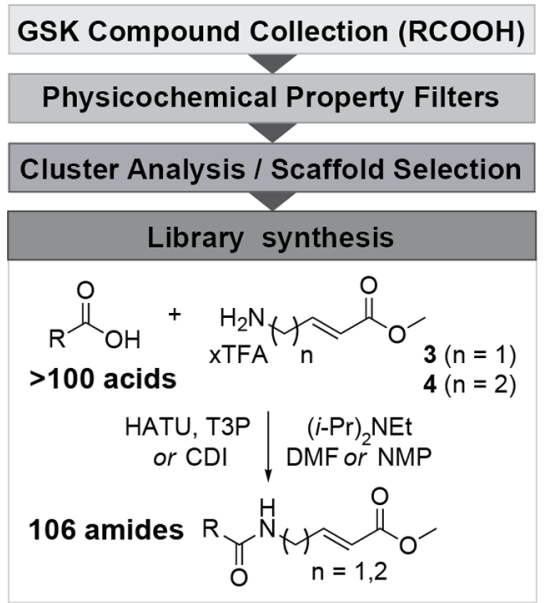

B

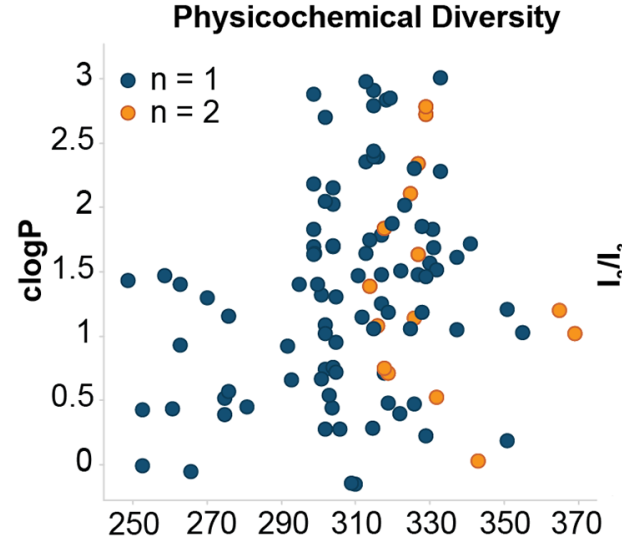

Molecular weight $(\mathrm{g} / \mathrm{mol})$
C

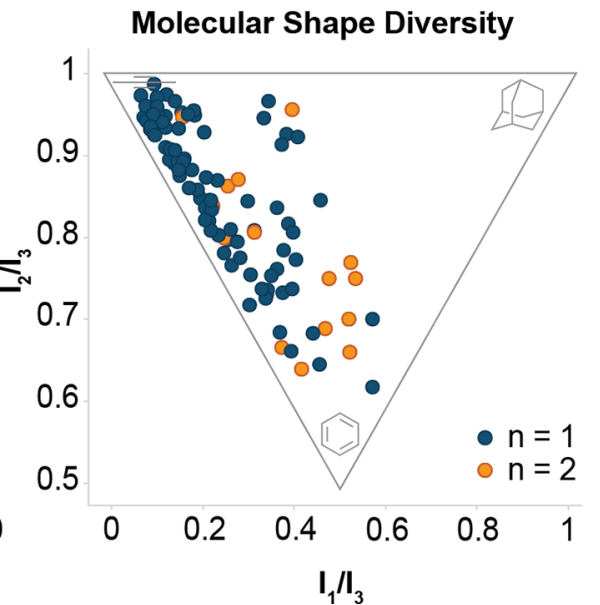

Figure 2. Design and synthesis of an electrophilic fragment library. (A) Parallel synthesis of the electrophilic fragment library from commercial and in-house sourced fragment carboxylic acids and the trifluoroacetic acid (TFA) salts of amines (3) and (4) by amide bond formation, using coupling reagents 1-[bis(dimethylamino)methylene]-1H-1,2,3-triazolo[4,5-b]pyridinium 3-oxide hexafluorophosphate (HATU), propylphosphonic anhydride (T3P), or $1,1^{\prime}$-carbonyldiimidazole (CDI). (B) The fragment library members clogP plotted against molecular weight illustrates the physicochemical diversity of the library. (C) The fragment library members normalized principal moment of inertia (PMI) were calculated in Molecular Operating Environment (MOE) ${ }^{33}$ based on low energy conformations and plotted as ratios in a triangular graph to illustrate the molecular shape diversity of the library. The vertices of this triangular plot $(0,1),(0.5,0.5)$, and $(1,1)$ represent a perfect rod, disc, and sphere, respectively.

modulators have been reported, including small molecule inhibitors BAY 11-7082 (1, Figure 1C) $)^{18}$ and gliotoxin (2), ${ }^{19}$ as well as stapled peptides that target protein-protein interactions at the HOIP/HOIL-1L and HOIL-1L/SHARPIN interfaces. $^{20-22}$ Although these modulators all have demonstrated effects on LUBAC activity, the molecules are also associated with drawbacks that may limit their utility as tools. Compound (1), which was originally identified as an inhibitor of $\mathrm{TNF} \alpha$-induced phosphorylation of $\mathrm{I} \kappa \mathrm{B} \alpha$ and NF- $\kappa \mathrm{B}$ signaling, has been shown to covalently inhibit HOIP activity yet exhibits broad reactivity across multiple proteins. ${ }^{18}$

Compound (2) is a well-characterized fungal metabolite and was recently identified in a high-throughput screen as an inhibitor of LUBAC. ${ }^{19}$ However, (2) interacts with multiple specific targets in mammalian cells, ${ }^{23,24}$ and as a complex natural product it does not easily lend itself to structure-based optimization. Stapled peptides suffer from similar disadvantages as staple type and position, and changes in peptide sequence can have profound effects on cell permeability. ${ }^{25-27}$ 
A

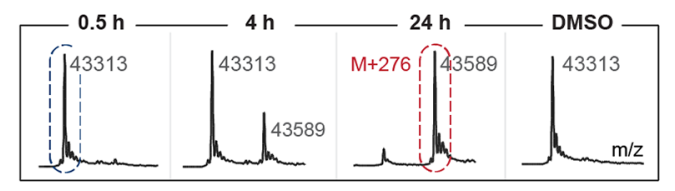

C

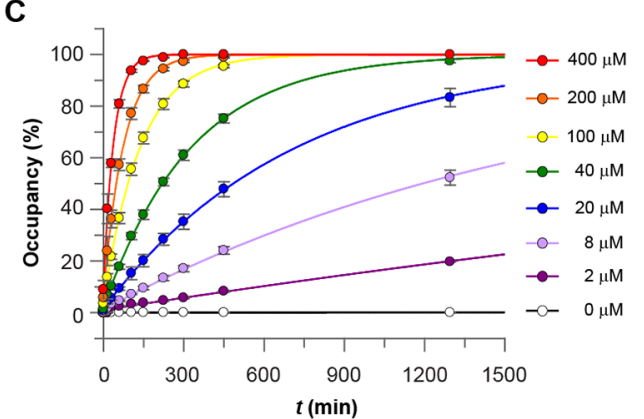

B

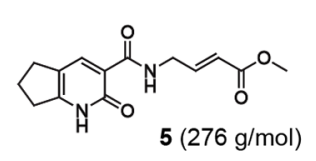

D

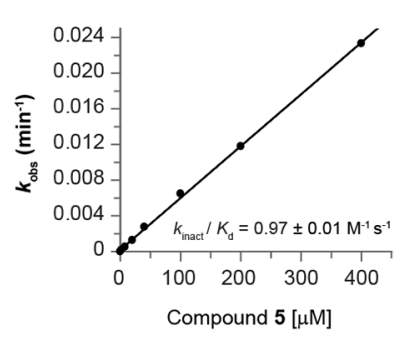

E

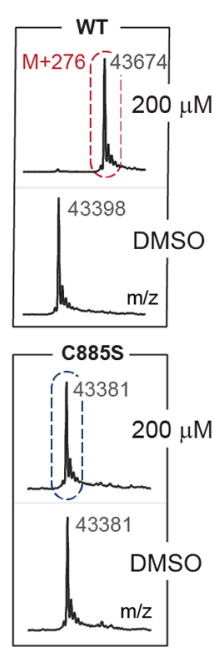

F

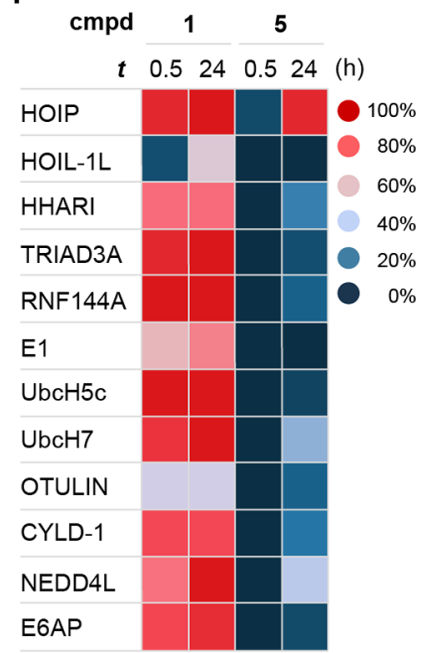

Figure 3. Compound (5) labels HOIP RBR at the active site cysteine residue C885 in a time- and concentration-dependent manner. (A) Compound (5) $(20 \mu \mathrm{M})$ labeled HOIP RBR R1032A (2 $\mu \mathrm{M})$ over time in the library screen. (B) The chemical structure of compound (5). (C) Labeling of wild type HOIP RBR $(2 \mu \mathrm{M})$ was validated by screening different concentrations of (5). Each time course was normalized to percentage occupancy, fitted to a single exponential equation, and plotted in GraFit (v.7) as an average of three independent experiments to determine the observed first-order rate constant $\left(k_{\mathrm{obs}}\right)$. (D) $k_{\mathrm{obs}}$ measurements were plotted against the concentration of $(5)$ to give the ratio of $k_{\text {inact }} / K_{\mathrm{d}}$ as the slope of the curve. ${ }^{34}$ (E) The active-site C885S mutant of HOIP RBR $(2 \mu \mathrm{M})$ was not labeled by compound (5) at $200 \mu \mathrm{M}$ after 4 h, suggesting that (5) targets the catalytic cysteine. (F) LC-MS based counter screen of compounds (1) (2-fold excess) and (5) (10-fold excess) against a panel of RBR E3 ligases (HOIL-1L, HHARI, TRIAD3A, RNF144A RBR domains), HECT E3 ligases (NEDD4L, E6AP), E2 enzymes (UbcH5c, UbcH7), E1 enzyme, and DUBs (OTULIN, CYLD-1). Data shown as percentage labeling after 0.5 and $24 \mathrm{~h}$ incubation.

Furthermore, a recent report describing a novel MALDI-TOF MS-based E2/E3 ligase screening assay identified the chemotherapeutic bendamustine as an inhibitor of HOIP RBR in vitro, albeit additional studies are warranted to verify its utility as a tool in a cellular setting. ${ }^{28}$

Inspired by recent reports describing the targeting of reactive cysteine residues in proteins using fragment-based covalent ligand screening of $\alpha, \beta$-unsaturated methyl esters, ${ }^{29,30}$ we decided to take a rational approach to target the active-site cysteine residue in HOIP as a means to covalently block its catalytic activity (Figure 1D). Herein, we describe the first inhibitors targeting the active-site cysteine of an RBR E3 ligase discovered by fragment-based covalent ligand screening and go on to show that such fragments serve as a useful starting point for structure-based development of selective, cell-permeable probes to interrogate the biology of LUBAC and other thioester-forming E3 ubiquitin ligases.

\section{RESULTS AND DISCUSSION}

Design and Synthesis of a Structurally Diverse Electrophilic Fragment Library. We synthesized a small library of fragments linked to $\alpha, \beta$-unsaturated methyl ester electrophiles by amide bond formation between carboxylic acid fragments, and amines (3) and (4) (Figure 2, Supporting Information, SI, Schemes S1 and S2). Compound collections based on the $\alpha, \beta$-unsaturated methyl ester scaffold (3) have been shown to exhibit a narrower reactivity profile compared to other commonly used electrophiles for covalent approaches, such as acrylamides. ${ }^{29,31,32}$ This advantageous reactivity profile promotes hit identification governed by molecular recognition rather than variations in the reactivity of the electrophile. ${ }^{29} \mathrm{We}$ chose to synthesize a small number of amides using the extended amine (4), based on the hypothesis that a longer linker between the fragment and electrophile may allow a greater sampling of potential protein interactions in the vicinity of the reactive cysteine residue. Fragments were selected from the GSK compound collection and commercial sources, based on physicochemical properties such as molecular weight, clogP, and hydrogen bond acceptors and donors (SI Figure S1 and Table S1), and clustered to ensure scaffold and functional group diversity. In total 106 amides were synthesized in parallel to give a physicochemically favorable and topologically diverse screening set of electrophilic fragments (Figure 2). A major concern of working with electrophilic compounds is their potential for poor stability. We monitored the DMSO stock solutions of the synthesized compounds over time to conclude that the vast majority were stable for $>6$ months in DMSO when stored at $4{ }^{\circ} \mathrm{C}$ (SI Figure S1). Furthermore, evaluation of the chemical stability of a representative set of compounds revealed good stability over a range of $\mathrm{pHs}$ and overall good resistance toward oxidation and glutathione trapping (SI Figure S1).

Identification of HOIP-Reactive Compounds by LCMS Screening. In total, 104 compounds were sufficiently stable in DMSO, and were pooled into 22 groups of 4-5 compounds (each compound separated in molecular weight by at least 5 mass units) and screened against the recombinant RBR domain of HOIP using protein LC-MS at a 10-fold stoichiometric excess of compound over protein $(20 \mu \mathrm{M}$ compound). Careful consideration of physicochemical properties during library design, and the adoption of a low compound screening concentration enabled a facile and robust LC-MS screening protocol with minimal sample handling and no need for protein purification prior to analysis. The initial library screen was carried out using HOIP RBR R1032A mutant protein, which behaves like wild type, ${ }^{15}$ while all further confirmatory experiments were carried out on the wild type HOIP RBR domain. Among the handful of preliminary hits that were identified (for full screening data see SI Figure S3), compound (5) stood out, producing $>85 \%$ labeling after $24 \mathrm{~h}$ 
A

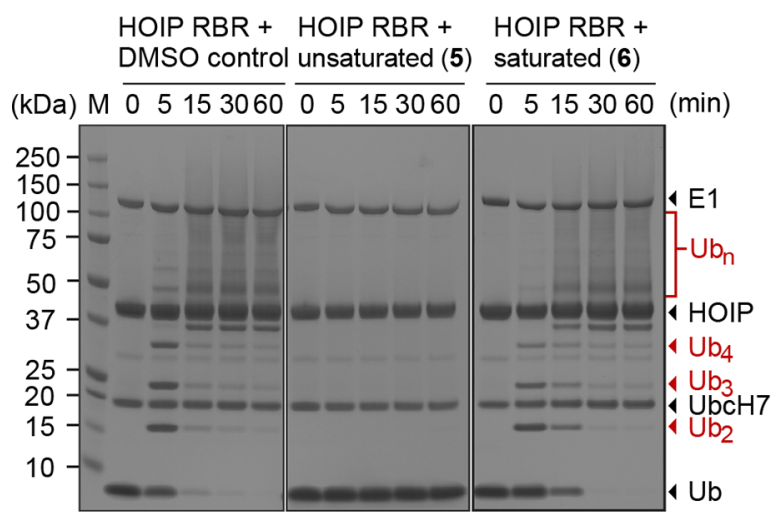

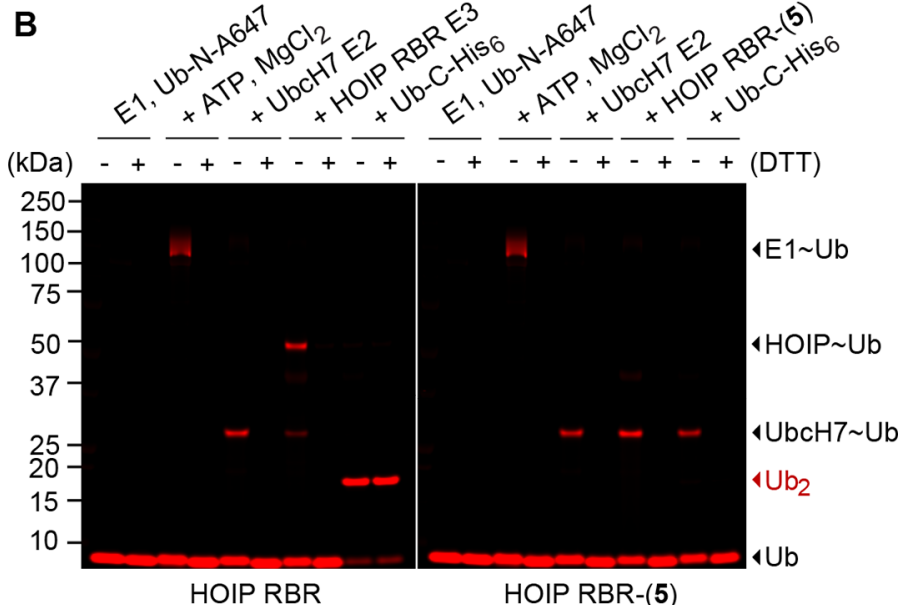

Figure 4. Compound (5) inhibits the formation of polyubiquitin chains by preventing loading of ubiquitin onto the HOIP RBR domain. (A) In vitro polyubiquitination assay monitoring the formation of unanchored polyubiquitin chains. Prelabeling of the HOIP RBR domain with compound (5) (middle) led to complete inhibition of polyubiquitin chain formation, whereas treatment with the saturated analogue (6) had no effect on catalytic activity. (B) In vitro ubiquitin transfer assay monitoring the sequential loading of N-terminally AlexaFluor647-labeled ubiquitin (Ub-NA647) onto E1, UbcH7, HOIP RBR domain, and a C-terminally His-tagged ubiquitin substrate (Ub-C-His 6 ). Prelabeling of the HOIP RBR domain with compound (5) (right) effectively prevented the loading of ubiquitin onto the E3, thereby inhibiting the formation of diubiquitin. For full gels see SI Figures S9 and S11.

incubation (Figure 3A-C). Incubation of HOIP RBR with compound (5) led to monolabeling of the protein in a timeand concentration-dependent manner as determined by LCMS. The concentration-response was analyzed according to a two-step model of reversible ligand binding followed by irreversible covalent modification $\left(\mathrm{P}+\mathrm{I} \rightleftarrows \mathrm{PI} \rightarrow \mathrm{PI}^{*}\right){ }^{34}$ Individual time courses were normalized to percentage occupancy and fitted to a single exponential equation to give $k_{\text {obs }}$ (Figure 3C) which was plotted against the concentration of compound (5) (Figure 3D). The observed relationship between $k_{\mathrm{obs}}$ and compound concentration was linear across the range of concentrations tested, which was limited by compound aqueous solubility, and it was therefore not possible to derive individual $k_{\text {inact }}$ and $K_{\mathrm{d}}$ values. Nonetheless, the gradient of the derivative plot gives the ratio $k_{\text {inact }} / K_{\mathrm{d}}(0.97 \pm$ $\left.0.01 \mathrm{M}^{-1} \mathrm{~s}^{-1}\right){ }^{34}$ We conclude that $K_{\mathrm{d}}$ is $>400 \mu \mathrm{M}$ for compound (5) and correspondingly, $k_{\text {inact }}$ will be $>0.0004 \mathrm{~s}^{-1}$ (Figure 3C, D and SI Figure S2). On the basis of the lower limit of $K_{\mathrm{d}}$ we can infer a ligand efficiency, LE $<0.24$ for compound (5). This relatively modest efficiency is in line with that observed for comparable ligands targeting the NEDD4-1 HECT E3 ligase. ${ }^{30}$ The LE may be reflective of the topology and available interactions in the binding site and should be contextualized as other HOIP inhibitors are reported. Treatment of the active-site mutant HOIP RBR C885S with compound (5) at a 100-fold stoichiometric excess did not result in protein labeling, strongly suggesting that compound (5) labels at the active site cysteine residue (Figure 3E). This finding was further corroborated by chymotryptic digest of the HOIP RBR-(5) adduct and LC-MS/MS analysis which unequivocally showed labeling on the catalytic residue C885 (SI Figure S5). Having successfully identified compound (5) as a covalent binder of the active site of the HOIP RBR E3 ligase, we sought to determine the specificity of this labeling. We performed an LC-MS-based counter screen of compound (5) against $\mathrm{E} 1$, the $\mathrm{E} 2 \mathrm{~s} \mathrm{UbcH} 5 \mathrm{c}$ and $\mathrm{UbcH} 7$, the RBR domains of the E3 ligases HOIL-1L, RNF144A, HHARI, and TRIAD3A, the HECT E3 ligases NEDD4L and E6AP, which all form covalent thioester intermediates with ubiquitin, and the two
DUBs known to cleave linear polyubiquitin chains OTULIN and CYLD-1 (Figure 3F). Compound (5) was screened at a 10-fold stoichiometric excess, and for comparison BAY 117082 (1) was used as a positive control at just 2 -fold excess. Unsurprisingly, compound (1) caused rapid and extensive labeling for most proteins while compound (5) displayed encouraging selectivity for HOIP, resulting in $<40 \%$ labeling after $24 \mathrm{~h}$ incubation for all other proteins screened (Figure 3F, SI Figure S6), indicating that compound (5) is a useful starting point for further ligand optimization.

HOIP-Reactive Hit Compound Inhibits Enzyme Function. The functional effects of HOIP labeling were investigated in an in vitro polyubiquitination assay. The HOIP RBR domain was preincubated with compound (5) to ensure full labeling and mixed with E1, E2 ( $\mathrm{UbcH} 7$ or $\mathrm{UbcH} 5 \mathrm{c}$ ), ubiquitin, and ATP to initiate unanchored polyubiquitin chain synthesis. Labeling of HOIP fully abolished chain formation when compared to DMSO control (Figure 4A, SI Figure S9). In contrast, treatment of HOIP with the saturated analog (6) (Scheme 1A) which is not able to form a covalent adduct had no inhibitory effect on polyubiquitin chain formation, demonstrating that the labeling occurs through the $\alpha, \beta$ unsaturated ester electrophile. Furthermore, a fluorescencebased ubiquitin transfer assay that monitors the sequential formation of E1, E2, and E3-ubiquitin thioesters, ${ }^{14}$ showed that labeling of the HOIP RBR domain with compound (5) inhibits formation of the $\mathrm{E} 3 \sim \mathrm{Ub}$ thioester intermediate, but not any of the previous steps, effectively blocking only ubiquitin transfer onto the protein substrate (Figure 4B).

Exploration of SAR through Targeted Compound Modifications. To investigate the structural determinants for compound recognition and labeling of the HOIP RBR domain we synthesized a set of closely related analogs, including saturated compounds (6) and (17), linker-extended analog (9), and fragment control (10) (Scheme 1A, D). Moreover, the pyridone scaffold was systematically altered to identify key protein-binding elements, including ring expansion (11a and b), aromatic rings $(\mathbf{1 1 c})$, changes to the hydrogen bond donor and acceptor abilities (11d-f), and fragment growth in 
Scheme 1. Analog Synthesis ${ }^{a}$

A

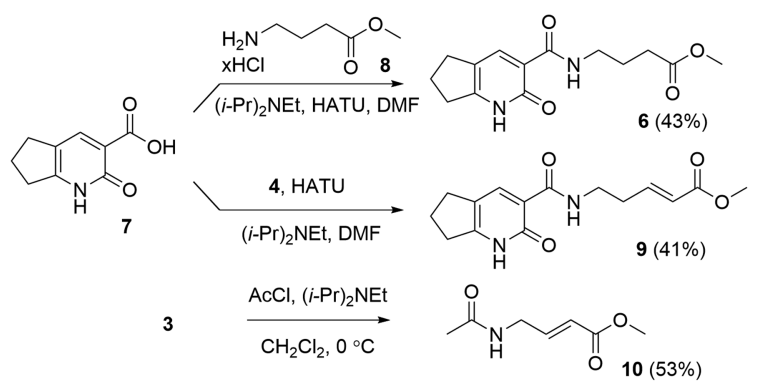

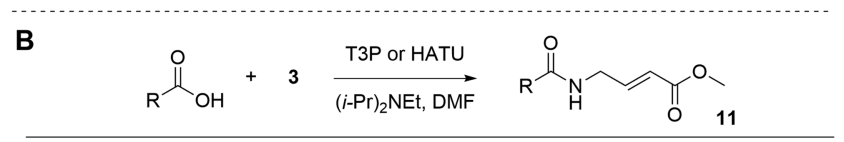
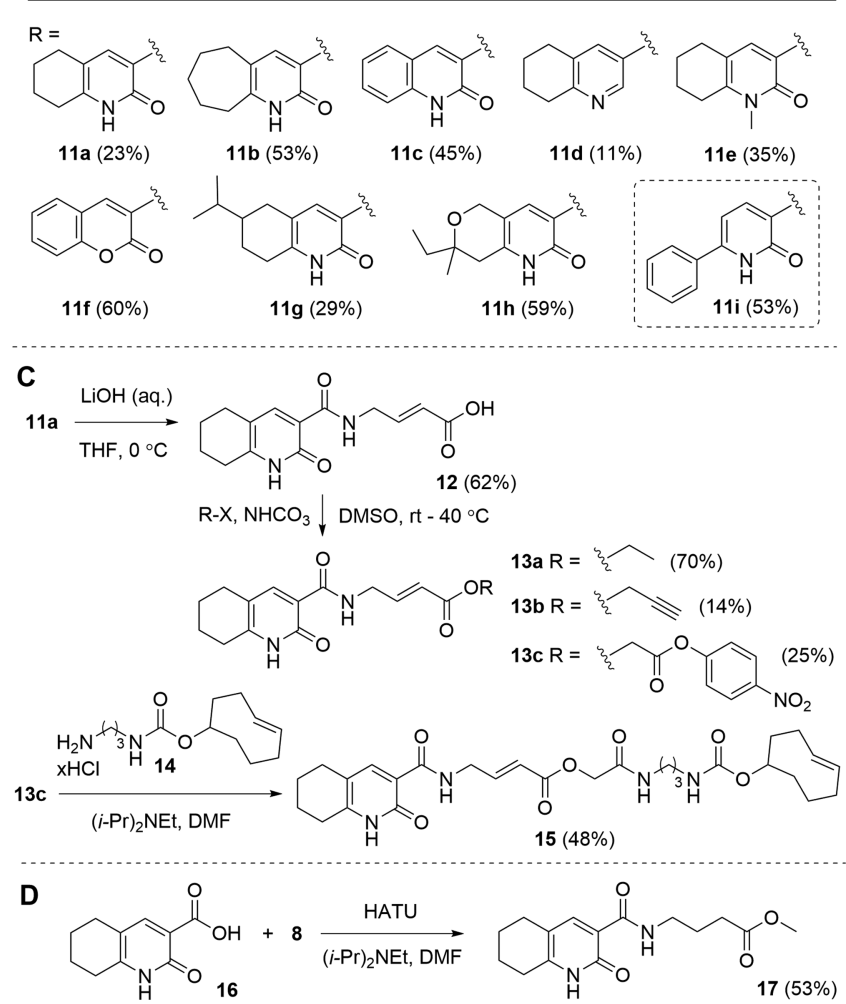

${ }^{a}$ (A) Control compounds (6), (9), and (10) were synthesized by amide bond formation using amines (8), (4), and (3) respectively. (B) Analogs $(11 \mathbf{a}-\mathbf{i})$ were synthesized by amide bond formation between the corresponding carboxylic acid and amine (3). Compound (11i) was synthesized as part of the original screening library. (C) Ester analogs $(\mathbf{1 3 a}-\mathbf{c})$ were synthesized in two steps from ester (11a) by alkaline hydrolysis and alkylation reactions using the appropriate alkyl halide (ethyl iodide, propargyl bromide, and 4nitrophenyl iodoacetate respectively), and (13c) was further converted into TCO probe (15). (D) Saturated control (17) was synthesized from carboxylic acid (16) and amine (8).

different vectors (11g-i; Scheme 1B). Compound (11i) was part of the original screening library. Lastly, we established a synthetic route based on sequential alkaline hydrolysis and alkylation to enable structural variation of the ester (13a and b), and the synthesis of a clickable trans-cyclooctene (TCO) probe (15) by amide bond formation between activated ester (13c), and amine (14; Scheme 1C). The analogs were screened by protein LC-MS against the HOIP RBR domain at a 10 -fold stoichiometric excess, and the percentage labeling was measured after $0.5,4$, and $24 \mathrm{~h}$ incubation (Figure 5A, SI
Figure S7). The data confirmed that saturation of the $\alpha, \beta$ unsaturated ester ( 6 and 17) abolished activity. Furthermore, it showed that extension of the linker between fragment and electrophile (9), or removal of the fragment portion of the molecule (10) resulted in significantly lower activity, suggesting that the fragment is essential for establishing key molecular interactions with the protein. The loss of reactivity observed when the hydrogen bonding ability of the pyridone was modified (e.g., analogs 11d-f) highlighted the importance of this group for binding and labeling. Alternative substitution patterns on the pyridone were more tolerated. Ring-expanded, and aromatic analogs $(11 \mathbf{a}-\mathbf{c})$ gave a comparable or increased rate of labeling, while fragment growth from different vectors of the pyridone (e.g., $11 \mathrm{~g}$ vs $11 \mathrm{~h}$ and $11 \mathrm{i}$ ), or growing the ester substituent (13a, 13b, and 15) had varying effects on activity, suggesting possible binding modes in the active site of the HOIP RBR domain for this chemical series.

Structural Analysis of the HOIP-Inhibitor Complex. To rationalize the observed reactivity of the analogs tested and to provide a basis for structure-based ligand optimization, we solved the crystal structure of the RING2-LDD domain of HOIP in covalent complex with inhibitor (5), at $2.15 \AA$ resolution (Figure 5B, SI Table S5). Compound (5) is bound within the active site of HOIP with clear contiguous electron density between $\mathrm{C} 885$ and the beta carbon of ester (5).

The cyclopentyl pyridone sits on a shallow ledge perfectly placed for its aromatic ring to stack above F888 and be anchored on one side by hydrogen bonds formed between the pyridone and the backbone nitrogen and oxygen of H889. This arrangement helps to explain why an extension of the linker between the pyridone and Michael acceptor in compound (9) led to reduced activity. Residues Y878, H889, and T891 enclose the other side of the ring system to form a defined, but probably somewhat malleable site, as demonstrated by the ability to accommodate small changes in ring sizes and substitutions in this region of the compound (e.g., 11a-c and 11g). Additional water-mediated and direct hydrogen bonds are made by all the other heteroatoms of (5) (Figure 5B). The ester chain of (5) lies within a pocket orthogonal to the ledge, with the carbonyl oxygen held in position by the hydroxyl side chain of S899, in a conformation that may facilitate the reaction of the catalytic cysteine and the Michael acceptor.

Sequence alignment of the RING2 domains of the RBR E3 ligases HOIP, HOIL-1L, and HHARI, TRIAD3A and RNF144A (Figure 5C) reveal sequence variation around the active-site cysteine, with Y878, F888, and S899 that contact the compound via their side chains not being conserved. This variation may account for some of the selectivity observed in labeling HOIP over other RBRs. Taken together, these observations suggest that specific molecular interactions between the fragment and protein help to position the electrophile favorably and promote sufficient residence time to support covalent bond formation.

Targeting of HOIP in Mammalian Cells. To provide further mechanistic characterization of the inhibitors we evaluated HOIP targeting in mammalian cells. For this purpose, we overexpressed the $3 \times$ FLAG-tagged HOIP RBR domain in HEK293T cells and used the slightly more potent analog (11a; Figure 5A). The cells were transiently transfected with $3 \times$ FLAG HOIP RBR for $48 \mathrm{~h}$, then incubated in media supplemented with inhibitor (11a) or inactive analogue (11i) at $30 \mu \mathrm{M}$ for $8 \mathrm{~h}$ before cell lysis. Following immunoprecipitation (IP) of the $3 \times$ FLAG-HOIP RBR domain with anti- 


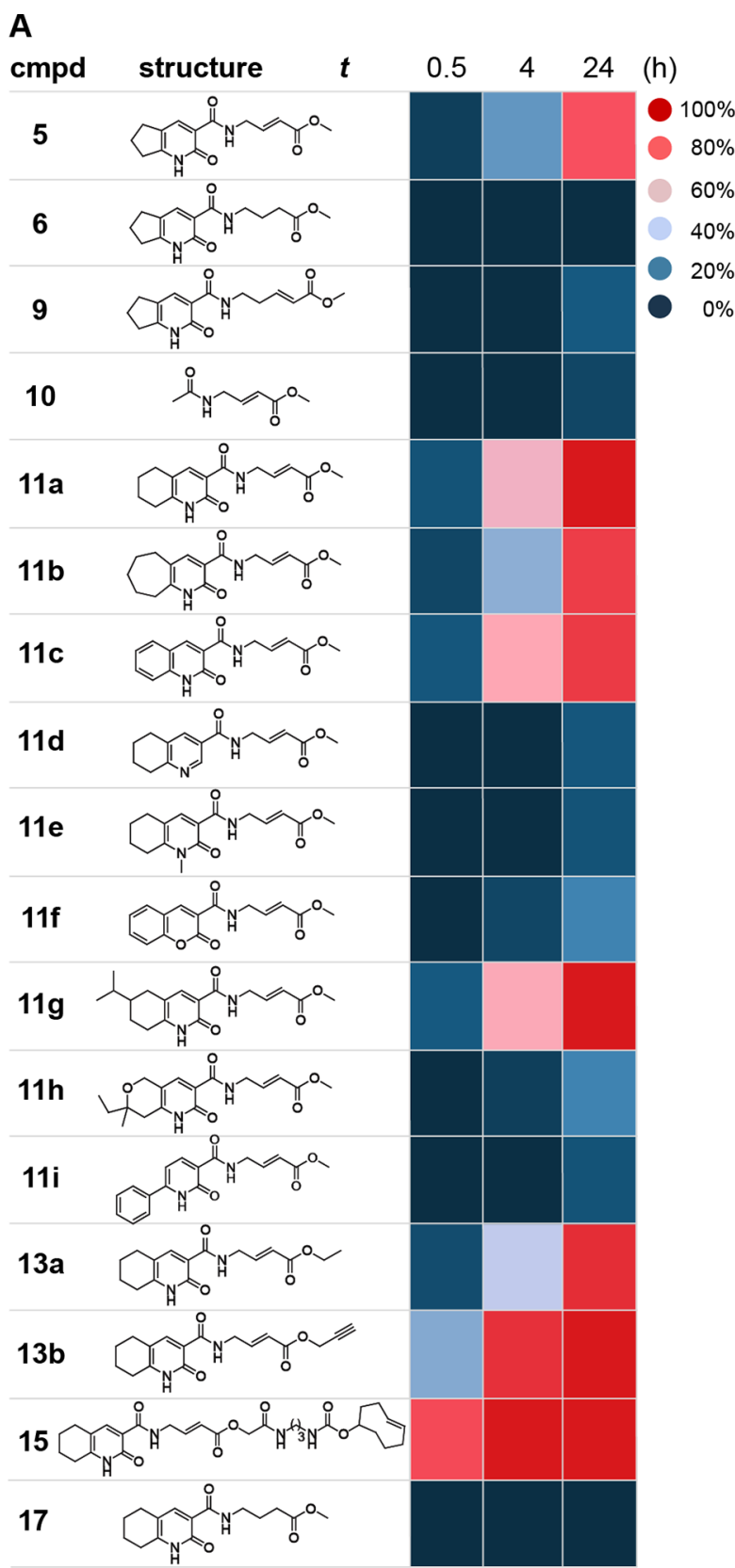

B

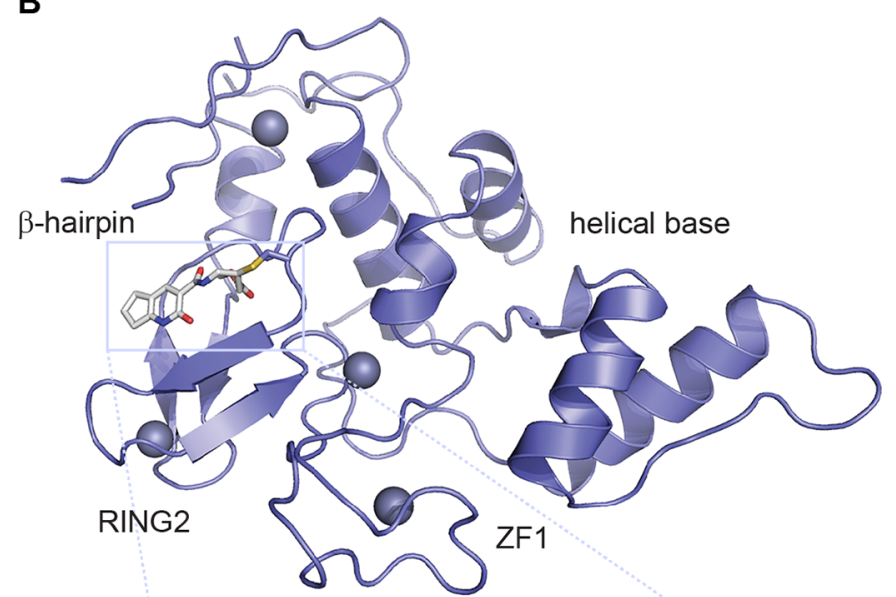

C

HOIP CPKCKFSY⿱艹 ALARGG-CMHFHCT--QCRHQFC $\stackrel{*}{\mathrm{~F}}$ HHARI

RNF144A

TRIAD3A HOIL-1L

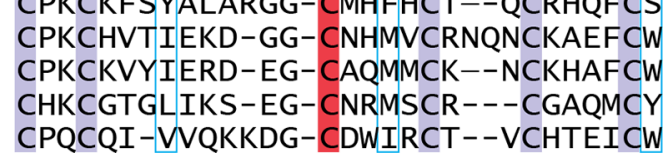

Figure 5. Molecular determinants for interactions between the HOIP RING2 domain and compound (5). (A) LC-MS screening data plotted as percentage labeling of the HOIP RBR domain $(2 \mu \mathrm{M})$ by key analogues (6), (9)-(11), (13), (15) $(20 \mu \mathrm{M})$, and $(\mathbf{1 7})(200 \mu \mathrm{M})$ after 0.5 , 4, and $24 \mathrm{~h}$ incubation. For full screening data see SI Figures S7 and S8. (B) Ribbon representation of the HOIP RING2-LDD domain structure in complex with compound (5) at $2.15 \AA$ A resolution (PDB: 6GZY). $\mathrm{Zn}^{2+}$ ions are shown as gray spheres and compound (5) as ball and stick. The inset shows a zoom into the active site showing hydrogen bonds (dashed lines) made by compound (5) with water molecules (red spheres) and HOIP (top), and the pocket around the active site which accommodates compound (5) (bottom). (C) Sequence alignment of RING2 domains of those RBR E3 ligases that were screened against compound (5). The catalytic cysteine residue is highlighted in red, noncatalytic cysteine residues in purple, and nonconserved residues contributing to the compound-binding pocket in HOIP are highlighted with a blue asterisk.

FLAG magnetic beads, the protein was digested on the beads with trypsin. Subsequent LC-MS/MS analysis showed that the HOIP RBR domain was significantly labeled by compound (11a) but not (11i) at C885, demonstrating that these inhibitors can effectively bind and label HOIP RBR in live cells (Figure 6A, B and SI Figure S12). Furthermore, immunoprecipitated $3 \times$ FLAG-tagged HOIP RBR (wild type and catalytically inactive C885A mutant) from HEK293T cells was used for an in vitro polyubiquitination assay with $\mathrm{E} 1, \mathrm{UbcH} 7$ and ubiquitin, to demonstrate that in-cell labeling of overexpressed HOIP RBR with compound (11a) leads to significant loss of the catalytic activity of HOIP, compared to (11i) and controls (Figure 6C). Given the central role of LUBAC in canonical NF- $\kappa$ B signaling, ${ }^{8}$ we then adopted an $N F-\kappa B$ Luciferase reporter assay to further demonstrate functional effects of HOIP inhibition. Treating HEK293T cells overexpressing full length HOIP, HOIL-1L, and SHARPIN with compound (11a) overnight led to inhibition of NF- $\kappa \mathrm{B}$ activation in a concentration-dependent manner, with an estimated $\mathrm{IC}_{50}$-value of $37 \pm 4 \mu \mathrm{M}$ (Figure $6 \mathrm{D}$, top, SI 
A

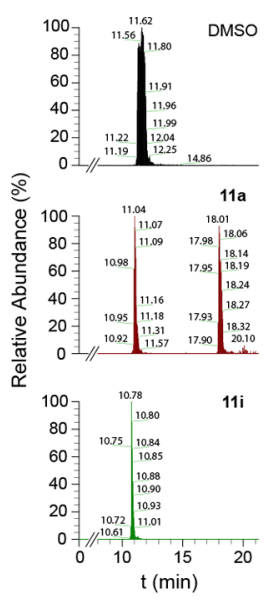

B

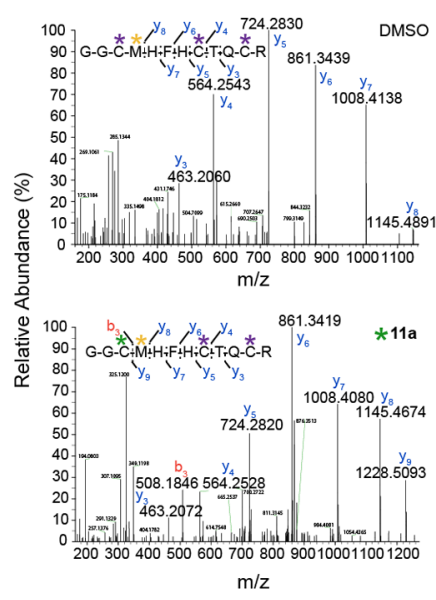

C

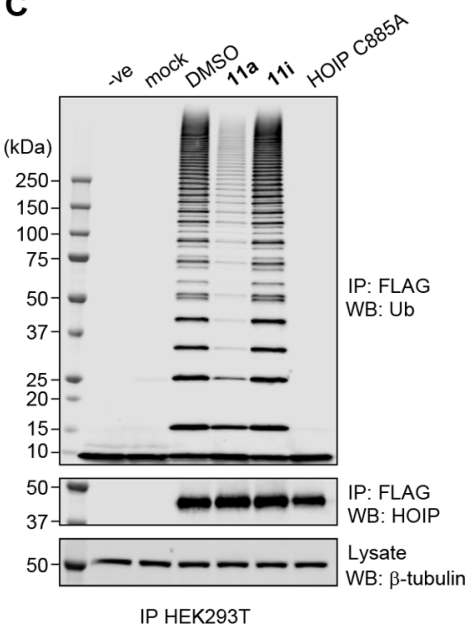

D

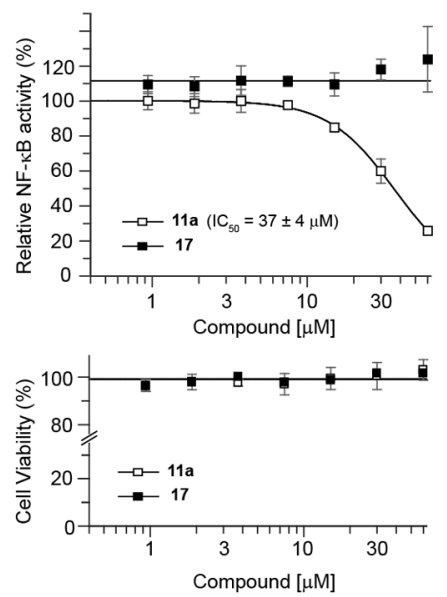

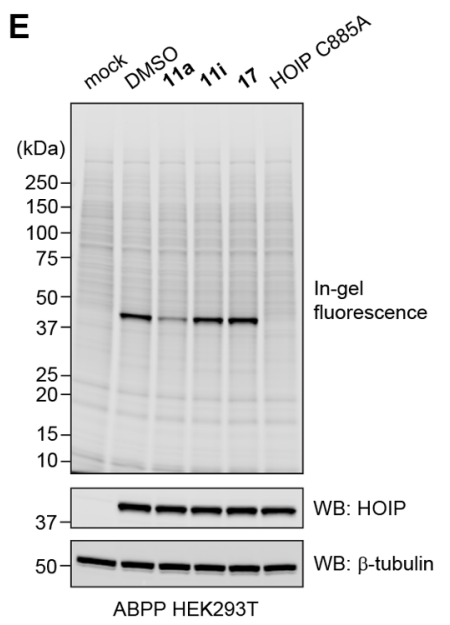

$\mathbf{F}$
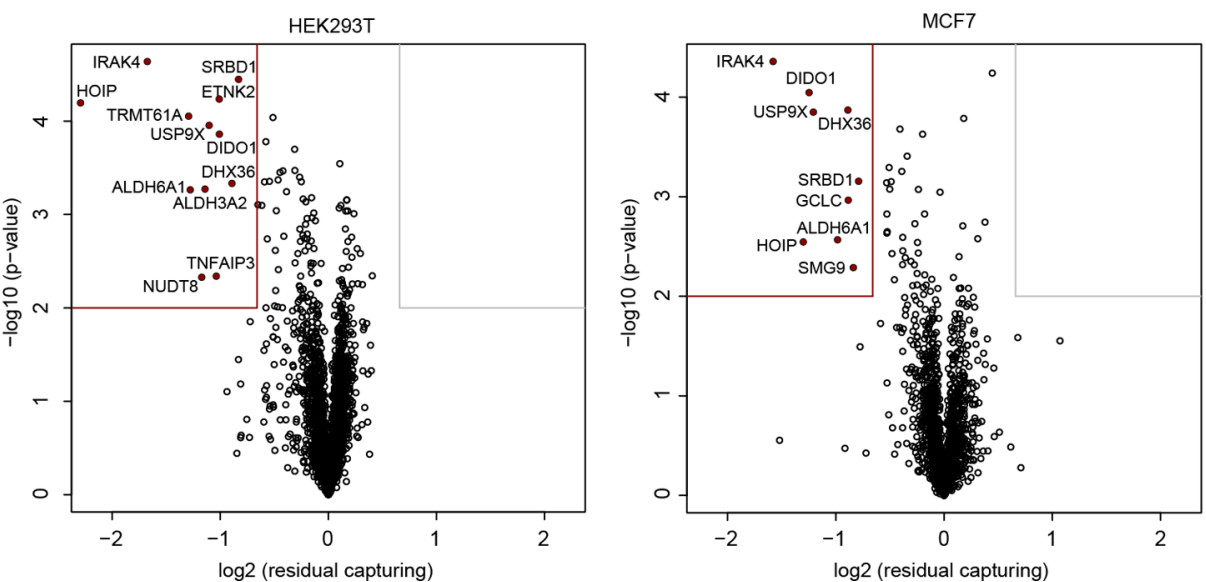

Figure 6. Compound (11a) labels and inhibits HOIP in HEK293T and MCF7 cells. (A) Total ion count traces of the dodecapeptide GGCMHFHCTQCR containing C885 after FLAG-IP and tryptic digest. Incubating HEK293T cells with compounds (11a) and (11i) at 30 $\mu$ M for $8 \mathrm{~h}$ gave significant labeling of overexpressed $3 \times$ FLAG-tagged HOIP RBR by compound (11a, middle) but not (11i, bottom). (B) LC-MS/ MS analysis of the GGCMHFHCTQCR peptides from DMSO control (top) and (11a)-treated cells (bottom), showing labeling of (11a) at C885 (green star). The samples were globally labeled with iodoacetamide (purple asterisk) prior to tryptic digest, and methionine oxidation (yellow asterisk) was taken into account. (C) On-bead polyubiquitination assay. In-cell labeling of overexpressed $3 \times$ FLAG-tagged HOIP RBR by compound (11a) $(30 \mu \mathrm{M})$ led to a significant decrease in free polyubiquitin chain formation compared to DMSO and $(11 \mathrm{i})(30 \mu \mathrm{M})$ controls. Non- (-ve), mock or $3 \times$ FLAG HOIP RBR C885A transfected HEK293T cells did not show formation of polyubiquitin chains in the polyubiquitin assay. The data are representative of two independent experiments. (D) NF- $\kappa$ B Luciferase reporter and CellTiter-Glo Luminescent cell viability (Promega) assays. Compound (11a) but not saturated analog (17) inhibited NF- $\kappa$ B activity in HEK293T cells overexpressing full-length HOIP, HOIL-1L, and SHARPIN in a dose-dependent manner (top), with no effect on cell viability (bottom). The graphs show average data of three independent experiments, each carried out in triplicate. (E) Activity-based protein profiling (ABPP) of HEK293T cells overexpressing $3 \times$ FLAGtagged HOIP RBR (wt and C885A mutant). Cells were preincubated with DMSO (control and HOIP C885A) or compounds (11a), (11i), or (17) $(30 \mu \mathrm{M})$ for $7 \mathrm{~h}$, then treated with TCO probe $(15)(3 \mu \mathrm{M})$ for $2 \mathrm{~h}$ and lysed. The cell lysates were treated with Cy5-Tz $(3 \mu \mathrm{M})$ for $1 \mathrm{~h}$. (F) ABPP of HEK293T (overexpressed HOIP RBR) and MCF7 cells (endogenous LUBAC expression), by competition of (15) (3 $\mu \mathrm{M})$ with (11a) $(30 \mu \mathrm{M})$, using quantitative MS. Specific labeling (maroon boxes) of HOIP (RNF31, SI datafile) was observed in both cell types. Treatments were carried out in triplicate. For full blots, see SI Figures S13 and S16.

Figure S14). Conversely, saturated analog (17) had no effect on NF- $\kappa$ B activity, and neither compound had any effect on cell viability (Figure 6D, bottom, SI Figure S14). Finally, we assessed the selectivity of compound (11a) on a proteomewide level using activity-based protein profiling (ABPP). ABPP is a powerful technique that utilizes covalent probes as handles for protein visualization or enrichment to evaluate protein engagement in cells, ${ }^{35}$ an approach that has recently enabled proteome-wide in-cell screening of covalent fragments. ${ }^{36,37} \mathrm{We}$ synthesized probe (15), based on the structure of (11a) and functionalized with a TCO moiety as enrichment handle to enable inverse-electron demand Diels-Alder (IED-DA) click chemistry with tetrazine $(\mathrm{Tz})$; IED-DA reactions typically give superior efficiency and selectivity for most protein labeling applications in cell lysates and in live cells compared to other types of click chemistries. ${ }^{38,39}$ In brief, HEK293T cells overexpressing $3 \times$ FLAG-tagged HOIP RBR domain were pretreated with (11a) followed by (15) before cell lysis. The impact of pretreatment time and concentration of (11a) and (15) was evaluated by in-gel fluorescence using Cy5-Tz (SI Figure S15). Pretreating the HEK293T cells with $30 \mu \mathrm{M}$ (11a) for $7 \mathrm{~h}$, then $3 \mu \mathrm{M}(\mathbf{1 5})$ for $2 \mathrm{~h}$, followed by lysis and TCO-Tz click reaction of the lysates gave significant reduction of the ingel fluorescence of HOIP RBR compared to (11i), (17), 
DMSO, and transfection controls (Figure 6E). Encouraged by these results, we then used quantitative MS to identify specifically labeled proteins. HEK293T cells overexpressing 3 $\times$ FLAG-tagged HOIP RBR, as well as MCF7 cells endogenously expressing LUBAC were treated with DMSO or (11a), then (15) as described. Proteins labeled by (15) were enriched using Tz-beads, ${ }^{38}$ treated with trypsin for onbead digest, and the resulting peptides were analyzed by quantitative MS. To ensure specificity, the residual capturing of every protein was calculated as log2-fold change competition versus DMSO control (mean ratio), $p$-values were calculated using student's $t$ test, and the results plotted so that specifically bound proteins with high $p$-value and strong fold-change populate the upper left quadrant (maroon boxes, Figure 6F). In addition to HOIP, 11 other proteins in HEK293T cells, and 8 other proteins in MCF7 cells were identified as being specifically covalently labeled by (11a). Among these proteins, IRAK4 is a kinase upstream of NF- $\kappa \mathrm{B}$ and therefore labeling by (11a) could impact physiological NF- $\kappa B$ signaling. However, in an IRAK4 substrate phosphorylation assay, compound (11a) showed no inhibition at concentrations up to $200 \mu \mathrm{M}$ (Supplementary Figure S17), indicating that it is unlikely to affect NF- $\kappa \mathrm{B}$ activation. Considering that no other RBR or HECT E3 ligases were identified as off-targets in the proteomics experiment, and that (11a) is an unoptimized fragment hit, the observed cellular selectivity profile is striking. Furthermore, specific labeling of HOIP in MCF7 cells provides the first evidence of targeting endogenous LUBAC in cells, showing that (11a) already constitutes a valuable tool compound and an excellent starting point for further optimization.

\section{CONCLUSIONS}

Despite the crucial biological roles of E3 ubiquitin ligases the development of selective tools that target their activity has been limited to date. Here, we describe how fragment-based covalent ligand screening allowed the rapid identification of inhibitors targeting the active site cysteine of the catalytic HOIP subunit of LUBAC, a member of the RBR family of E3s. A diverse library of fragments linked to $\alpha, \beta$-unsaturated ester electrophiles was synthesized and rapidly screened by protein LC-MS to identify compound (5) as a covalent binder of HOIP. The established screening protocol required minimal sample handling and enabled expedient screening of multiple proteins. Biochemical characterization of (5) and its analog (11a) demonstrated that these compounds label HOIP with promising proteome-wide selectivity and effectively inhibit linear polyubiquitin chain formation in vitro and in a cellular environment. The combination of analog synthesis and a highresolution crystal structure of the HOIP RING2-LDD domain in covalent complex with $(\mathbf{5})$ provides the first insight into the molecular requirements for fragment recognition by HOIP and will allow the rational design of inhibitors with increased activity and specificity for this RBR family member. Taken together, our results show the first proof-of-concept for screening and rational design of a covalent inhibitor library designed to target the active site of an RBR E3 ligase, an approach which is generally applicable to other thioesterforming E3 ligases and should help in the search for novel inhibitors of this important class of enzymes.

\section{ASSOCIATED CONTENT}

\section{Supporting Information}

The Supporting Information is available free of charge on the ACS Publications website at DOI: 10.1021/jacs.8b13193.

Detailed synthetic procedures for all synthesized compounds, ${ }^{1} \mathrm{H}$ NMR spectra and LC-MS chromatograms for all library compounds, ${ }^{1} \mathrm{H},{ }^{13} \mathrm{C}$ NMR, LC-MS and HRMS data for all synthesized hit analogs, physicochemical analyses of the screening library, details of recombinant protein expression and purification, experimental procedures for biochemical assays, TOF LC-MS protocol and protein deconvolution data for LC-MS-based compound screening, protein X-ray crystallography, ABPP, and cell based experimenst (PDF)

Datafile for the quantitative MS-based ABPP experiment (XLSX)

\section{AUTHOR INFORMATION}

\section{Corresponding Authors}

*katrin.rittinger@crick.ac.uk

*david.x.house@gsk.com

ORCID

Henrik Johansson: 0000-0002-9990-6261

Marcel Muelbaier: 0000-0002-5414-0258

David House: 0000-0002-9635-2451

Katrin Rittinger: 0000-0002-7698-4435

\section{Notes}

The authors declare no competing financial interest.

\section{ACKNOWLEDGMENTS}

We thank Andy Purkiss from the Crick Structural Biology Technology Platform for help with data processing and crystallographic analysis, the Diamond Light Source, Oxford, U.K. for synchrotron access, Abigail Davies and Ashleigh Howes for the $3 \times$ FLAG HOIP RBR pcDNA3.1(+) construct, Georgina Green for managing compound DMSO stock solutions and carrying out the DMSO stability LC-MS assays, Ruben Gomez-Sanchez and Natalie Warren-Godkin for carrying out the chemical stability assays, and Shenaz Bunally for determining kinetic solubility and ChromLogD7.4 for the representative subset of the electrophilic fragment library. We thank S. Lehmann for assay support and M. Boesche, T. Rudi, M. Kloes-Hudak, and K. Kammerer for assistance with mass spectrometry. Henning Walczak (HOIP, HOIL-1L and SHARPIN) and Teresa Thurston (pRDII Luc) are thanked for their kind donation of plasmids for the NF- $\kappa \mathrm{B}$ assay. Thau F. Ho is thanked for the expression and purification of IRAK4 protein. Sean Lynn is thanked for assistance with NMR, and Marcela Castro for assistance with manuscript figure design and preparation. We thank Andy Powell and David Powell for helpful discussions. This work was supported by the Francis Crick Institute which receives its core funding from Cancer Research U.K. (FC001142 to K.R.), the U.K. Medical Research Council (FC001142 to K.R.) and the Wellcome Trust (FC001142 to K.R.). We also thank GSK for its commitment to support fundamental discovery research through the establishment of the Crick-GSK LinkLabs partnership. 


\section{REFERENCES}

(1) Komander, D.; Rape, M. The ubiquitin code. Annu. Rev. Biochem. 2012, 81, 203-29.

(2) Pickart, C. M.; Eddins, M. J. Ubiquitin: structures, functions, mechanisms. Biochim. Biophys. Acta, Mol. Cell Res. 2004, 1695 (1-3), $55-72$.

(3) Cohen, P.; Tcherpakov, M. Will the Ubiquitin System Furnish as Many Drug Targets as Protein Kinases? Cell 2010, 143 (5), 686-693.

(4) Huang, X.; Dixit, V. M. Drugging the undruggables: exploring the ubiquitin system for drug development. Cell Res. 2016, 26 (4), 484-98.

(5) Kirisako, T.; Kamei, K.; Murata, S.; Kato, M.; Fukumoto, H.; Kanie, M.; Sano, S.; Tokunaga, F.; Tanaka, K.; Iwai, K. A ubiquitin ligase complex assembles linear polyubiquitin chains. EMBO J. 2006, 25 (20), 4877-87.

(6) Tokunaga, F.; Sakata, S.; Saeki, Y.; Satomi, Y.; Kirisako, T.; Kamei, K.; Nakagawa, T.; Kato, M.; Murata, S.; Yamaoka, S.; Yamamoto, M.; Akira, S.; Takao, T.; Tanaka, K.; Iwai, K. Involvement of linear polyubiquitylation of NEMO in NF-kappaB activation. Nat. Cell Biol. 2009, 11 (2), 123-32.

(7) Rieser, E.; Cordier, S. M.; Walczak, H. Linear ubiquitination: a newly discovered regulator of cell signalling. Trends Biochem. Sci. 2013, 38 (2), 94-102.

(8) Sasaki, K.; Iwai, K. Roles of linear ubiquitinylation, a crucial regulator of NF-kappaB and cell death, in the immune system. Immunol. Rev. 2015, 266 (1), 175-89.

(9) Rittinger, K.; Ikeda, F. Linear ubiquitin chains: enzymes, mechanisms and biology. Open Biol. 2017, 7 (4), 170026

(10) Hrdinka, M.; Gyrd-Hansen, M. The Met1-Linked Ubiquitin Machinery: Emerging Themes of (De)regulation. Mol. Cell 2017, 68 (2), 265-280.

(11) Ikeda, F.; Deribe, Y. L.; Skanland, S. S.; Stieglitz, B.; Grabbe, C.; Franz-Wachtel, M.; van Wijk, S. J.; Goswami, P.; Nagy, V.; Terzic, J.; Tokunaga, F.; Androulidaki, A.; Nakagawa, T.; Pasparakis, M.; Iwai, K.; Sundberg, J. P.; Schaefer, L.; Rittinger, K.; Macek, B.; Dikic, I. SHARPIN forms a linear ubiquitin ligase complex regulating NFkappaB activity and apoptosis. Nature 2011, 471 (7340), 637-41.

(12) Gerlach, B.; Cordier, S. M.; Schmukle, A. C.; Emmerich, C. H.; Rieser, E.; Haas, T. L.; Webb, A. I.; Rickard, J. A.; Anderton, H.; Wong, W. W.; Nachbur, U.; Gangoda, L.; Warnken, U.; Purcell, A. W.; Silke, J.; Walczak, H. Linear ubiquitination prevents inflammation and regulates immune signalling. Nature 2011, 471 (7340), 591-6.

(13) Tokunaga, F.; Nakagawa, T.; Nakahara, M.; Saeki, Y.; Taniguchi, M.; Sakata, S.; Tanaka, K.; Nakano, H.; Iwai, K. SHARPIN is a component of the NF-kappaB-activating linear ubiquitin chain assembly complex. Nature 2011, 471 (7340), 633-6.

(14) Stieglitz, B.; Morris-Davies, A. C.; Koliopoulos, M. G.; Christodoulou, E.; Rittinger, K. LUBAC synthesizes linear ubiquitin chains via a thioester intermediate. EMBO Rep. 2012, 13 (9), 840-6.

(15) Stieglitz, B.; Rana, R. R.; Koliopoulos, M. G.; Morris-Davies, A. C.; Schaeffer, V.; Christodoulou, E.; Howell, S.; Brown, N. R.; Dikic, I.; Rittinger, K. Structural basis for ligase-specific conjugation of linear ubiquitin chains by HOIP. Nature 2013, 503 (7476), 422-426.

(16) Smit, J. J.; Monteferrario, D.; Noordermeer, S. M.; van Dijk, W. J.; van der Reijden, B. A.; Sixma, T. K. The E3 ligase HOIP specifies linear ubiquitin chain assembly through its RING-IBR-RING domain and the unique LDD extension. EMBO J. 2012, 31 (19), 3833-44.

(17) Lechtenberg, B. C.; Rajput, A.; Sanishvili, R.; Dobaczewska, M. K.; Ware, C. F.; Mace, P. D.; Riedl, S. J. Structure of a HOIP/E2 ubiquitin complex reveals RBR E3 ligase mechanism and regulation. Nature 2016, 529 (7587), 546-50.

(18) Strickson, S.; Campbell, D. G.; Emmerich, C. H.; Knebel, A.; Plater, L.; Ritorto, M. S.; Shpiro, N.; Cohen, P. The anti-inflammatory drug BAY 11-7082 suppresses the MyD88-dependent signalling network by targeting the ubiquitin system. Biochem. J. 2013, 451 (3), 427-37.

(19) Sakamoto, H.; Egashira, S.; Saito, N.; Kirisako, T.; Miller, S.; Sasaki, Y.; Matsumoto, T.; Shimonishi, M.; Komatsu, T.; Terai, T.; Ueno, T.; Hanaoka, K.; Kojima, H.; Okabe, T.; Wakatsuki, S.; Iwai,
K.; Nagano, T. Gliotoxin suppresses NF-kappaB activation by selectively inhibiting linear ubiquitin chain assembly complex (LUBAC). ACS Chem. Biol. 2015, 10 (3), 675-81.

(20) Aguilar-Alonso, F.; Whiting, A. L.; Kim, Y. J.; Bernal, F. Biophysical and biological evaluation of optimized stapled peptide inhibitors of the linear ubiquitin chain assembly complex (LUBAC). Bioorg. Med. Chem. 2018, 26 (6), 1179-1188.

(21) Yang, Y.; Schmitz, R.; Mitala, J.; Whiting, A.; Xiao, W.; Ceribelli, M.; Wright, G. W.; Zhao, H.; Yang, Y.; Xu, W.; Rosenwald, A.; Ott, G.; Gascoyne, R. D.; Connors, J. M.; Rimsza, L. M.; Campo, E.; Jaffe, E. S.; Delabie, J.; Smeland, E. B.; Braziel, R. M.; Tubbs, R. R.; Cook, J. R.; Weisenburger, D. D.; Chan, W. C.; Wiestner, A.; Kruhlak, M. J.; Iwai, K.; Bernal, F.; Staudt, L. M. Essential role of the linear ubiquitin chain assembly complex in lymphoma revealed by rare germline polymorphisms. Cancer Discovery 2014, 4 (4), 480-93.

(22) Fujita, H.; Tokunaga, A.; Shimizu, S.; Whiting, A. L.; AguilarAlonso, F.; Takagi, K.; Walinda, E.; Sasaki, Y.; Shimokawa, T.; Mizushima, T.; Ohki, I.; Ariyoshi, M.; Tochio, H.; Bernal, F.; Shirakawa, M.; Iwai, K. Cooperative Domain Formation by Homologous Motifs in HOIL-1L and SHARPIN Plays A Crucial Role in LUBAC Stabilization. Cell Rep. 2018, 23 (4), 1192-1204.

(23) Waring, P.; Beaver, J. Gliotoxin and related epipolythiodioxopiperazines. Gen. Pharmacol. 1996, 27 (8), 1311-6.

(24) Dolan, S. K.; O’Keeffe, G.; Jones, G. W.; Doyle, S. Resistance is not futile: gliotoxin biosynthesis, functionality and utility. Trends Microbiol. 2015, 23 (7), 419-28.

(25) Chu, Q. M.; Moellering, R. E.; Hilinski, G. J.; Kim, Y.-W.; Grossmann, T. N.; Yeh, J. T.-H.; Verdine, G. L. Towards understanding cell penetration by stapled peptides. MedChem Comm 2015, 6 (1), 111-119.

(26) Walensky, L. D.; Bird, G. H. Hydrocarbon-stapled peptides: principles, practice, and progress. J. Med. Chem. 2014, 57 (15), 627588.

(27) Cromm, P. M.; Spiegel, J.; Grossmann, T. N. Hydrocarbon stapled peptides as modulators of biological function. ACS Chem. Biol. 2015, 10 (6), 1362-75.

(28) De Cesare, V.; Johnson, C.; Barlow, V.; Hastie, J.; Knebel, A.; Trost, M. The MALDI-TOF E2/E3 Ligase Assay as Universal Tool for Drug Discovery in the Ubiquitin Pathway. Cell Chem. Biol. 2018, 25 (9), 1117-1127.

(29) Kathman, S. G.; Xu, Z.; Statsyuk, A. V. A Fragment-Based Method to Discover Irreversible Covalent Inhibitors of Cysteine Proteases. J. Med. Chem. 2014, 57 (11), 4969-4974.

(30) Kathman, S. G.; Span, I.; Smith, A. T.; Xu, Z.; Zhan, J.; Rosenzweig, A. C.; Statsyuk, A. V. A Small Molecule That Switches a Ubiquitin Ligase From a Processive to a Distributive Enzymatic Mechanism. J. Am. Chem. Soc. 2015, 137 (39), 12442-5.

(31) Flanagan, M. E.; Abramite, J. A.; Anderson, D. P.; Aulabaugh, A.; Dahal, U. P.; Gilbert, A. M.; Li, C.; Montgomery, J.; Oppenheimer, S. R.; Ryder, T.; Schuff, B. P.; Uccello, D. P.; Walker, G. S.; Wu, Y.; Brown, M. F.; Chen, J. M.; Hayward, M. M.; Noe, M. C.; Obach, R. S.; Philippe, L.; Shanmugasundaram, V.; Shapiro, M. J.; Starr, J.; Stroh, J.; Che, Y. Chemical and computational methods for the characterization of covalent reactive groups for the prospective design of irreversible inhibitors. J. Med. Chem. 2014, 57 (23), 10072-9.

(32) Cee, V. J.; Volak, L. P.; Chen, Y.; Bartberger, M. D.; Tegley, C.; Arvedson, T.; McCarter, J.; Tasker, A. S.; Fotsch, C. Systematic Study of the Glutathione (GSH) Reactivity of N-Arylacrylamides: 1. Effects of Aryl Substitution. J. Med. Chem. 2015, 58 (23), 9171-8.

(33) Molecular Operating Environment (MOE), C. C. G. U., 1010 Sherbooke St. West, Suite \#910, Montreal, QC, Canada, H3A 2R7 2018.

(34) Strelow, J. M. A Perspective on the Kinetics of Covalent and Irreversible Inhibition. SLAS Discov 2017, 22 (1), 3-20.

(35) Heal, W. P.; Dang, T. H.; Tate, E. W. Activity-based probes: discovering new biology and new drug targets. Chem. Soc. Rev. 2011, 40 (1), 246-57. 
(36) Backus, K. M.; Correia, B. E.; Lum, K. M.; Forli, S.; Horning, B. D.; Gonzalez-Paez, G. E.; Chatterjee, S.; Lanning, B. R.; Teijaro, J. R.; Olson, A. J.; Wolan, D. W.; Cravatt, B. F. Proteome-wide covalent ligand discovery in native biological systems. Nature 2016, 534 (7608), 570-574.

(37) Parker, C. G.; Galmozzi, A.; Wang, Y.; Correia, B. E.; Sasaki, K.; Joslyn, C. M.; Kim, A. S.; Cavallaro, C. L.; Lawrence, R. M.; Johnson, S. R.; Narvaiza, I.; Saez, E.; Cravatt, B. F. Ligand and Target Discovery by Fragment-Based Screening in Human Cells. Cell 2017, 168 (3), 527-541.

(38) Rutkowska, A.; Thomson, D. W.; Vappiani, J.; Werner, T.; Mueller, K. M.; Dittus, L.; Krause, J.; Muelbaier, M.; Bergamini, G.; Bantscheff, M. A Modular Probe Strategy for Drug Localization, Target Identification and Target Occupancy Measurement on Single Cell Level. ACS Chem. Biol. 2016, 11 (9), 2541-50.

(39) Murrey, H. E.; Judkins, J. C.; Am Ende, C. W.; Ballard, T. E.; Fang, Y.; Riccardi, K.; Di, L.; Guilmette, E. R.; Schwartz, J. W.; Fox, J. M.; Johnson, D. S. Systematic Evaluation of Bioorthogonal Reactions in Live Cells with Clickable HaloTag Ligands: Implications for Intracellular Imaging. J. Am. Chem. Soc. 2015, 137 (35), 11461-75. 